\title{
Sjögren's syndrome - an update for dental practitioners
}

\author{
A. J. Carr, ${ }^{1}$ W.-F. Ng, ${ }^{2}$ F. Figueiredo, ${ }^{3}$ R. I. Macleod, ${ }^{4}$ M. Greenwood ${ }^{5}$ \\ and K. Staines ${ }^{6}$
}

VERIFIABLE CPD PAPER

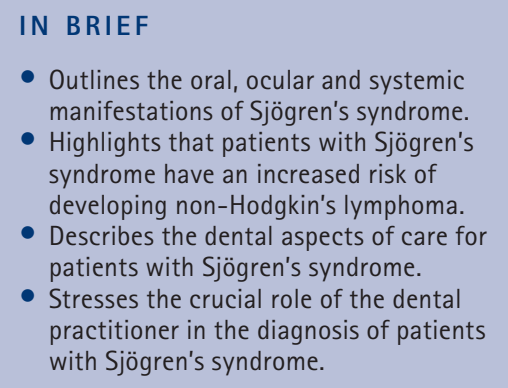

Sjögren's syndrome (SS), an autoimmune, multi-factorial disorder, affects around $5 \%$ of females and $0.5 \%$ of males in the general population. The dental practitioner has a key role in recognising the clinical features of this condition, organising referral for specialist care and managing the oral health of these patients. In this article, we summarise the clinical manifestations, diagnosis and management of SS relevant to dental practitioners.

\section{INTRODUCTION}

Sjögren's Syndrome (SS) is an autoimmune disorder which affects around 0.5\% of adults, with prevalence increasing with age and a female to male ratio of 9:1. ${ }^{1}$ The cardinal symptoms of SS is a dry mouth (xerostomia), together with dryness of the eyes (keratoconjunctivitis sicca). ${ }^{1}$ Primary Sjögren's syndrome (PSS) is a systemic autoimmune disorder characterised by inflammation of the exocrine glands, such as lacrimal and salivary glands without any associated connective tissue disease. PSS ultimately results in hypofunction and dryness of the mucosal surfaces, particularly of the eyes and mouth. ${ }^{2}$

Secondary Sjögren's syndrome (SSS) has similar pathophysiology, signs and symptoms as PSS, but is associated with some forms of connective tissue disease such as

\footnotetext{
${ }^{1 *}$ Academic Clinical Fellow, Department of Dental and Maxillofacial Radiology, Newcastle Dental Hospital, Newcastle upon Tyne; ${ }^{2}$ Clinical Senior Lecturer and Honourary Consultant in Rheumatology, Musculoskeletal Research Group, Institute of Cellular Medicine, Faculty of Medical Sciences, Newcastle University, Framlington Place, Newcastle upon Tyne; ${ }^{3}$ Consultant Ophthalmologist and Clinical Senior Lecturer, Department of Ophthalmology, Royal Victoria Infirmary, Newcastle upon Tyne; ${ }^{4}$ Consultant and Honourary Clinical Senior Lecturer, Department of Dental and Maxillofacial Radiology, Newcastle Dental Hospital, Newcastle upon Tyne; ${ }^{5}$ FHEA Consultant and Honourary Clinical Professor in Oral and Maxillofacial Surgery, Department of Oral and Maxillofacial Surgery, Newcastle Dental Hospital, Newcastle upon Tyne; ${ }^{6}$ Consultant in Oral Medicine, Department of Oral Medicine, Newcastle Dental Hospital, Newcastle upon Tyne

* Correspondence to: Dr Andrew Carr

Email: andrew.carr2@ncl.ac.uk
}

\section{Refereed Paper}

Accepted 5 September 2012

DOI: $10.1038 /$ sj.bdj.2012.890

${ }^{\circledR}$ British Dental Journal 2012; 213: 353-357 rheumatoid arthritis and systemic lupus erythematosus. Primary biliary cirrhosis and other autoimmune conditions can also be associated with SSS.

It is important to recognise that as SS is a systemic disease; patients can often suffer from disabling fatigue, skin lesions, haematological problems such as anaemia, vulval dryness and gastrointestinal complaints. ${ }^{3}$ Sjögren's Syndrome was originally reported by Henrik Sjögren in $1930 .^{4}$

\section{ORAL MANIFESTATIONS}

The predominant effects of SS on the oral cavity are mainly consequent to hyposalivation. Recent evidence indicates that over half of the patients with PSS experienced an oral symptom as their first manifestation of the condition. ${ }^{2}$

However, PSS can also indirectly cause oral manifestations secondary to systemic involvement. An appropriate example is that of SS-induced thrombocytopenia manifesting as oral bruising or purpura. Significant thrombocytopenia at levels below $50 \times 10^{9}$ may also influence any planned dental treatment.

PSS patients are 44 times more likely to develop B cell lymphomas of the salivary gland. ${ }^{5}$ The presence of any firm or discrete swelling involving major salivary glands or presenting intraorally should raise suspicion and may warrant further investigation.

Lack of saliva causes difficulties in oral function. Patients may complain that their lips stick together and they have difficulties eating, speaking, chewing, swallowing and with denture retention. ${ }^{6,7}$

Saliva may be of a frothy consistency with an absence of saliva pooling in the floor of mouth. It may not be possible to express saliva from the parotid and submandibular ducts. The tongue may appear erythematous, dry and fissured. Alternatively the tongue may be coated and appear brown or black, a presentation appropriately termed black hairy tongue. The oral mucosa may appear dry and stick to the dental mirror or gloved finger, have a normal appearance or appear erythematous. Such dry erythematous mucosa is often uncomfortable to touch with patients complaining of soreness even in the absence of overt clinical infection. ${ }^{8}$ Patients may also complain of dysguesia and halitosis. Oral candidosis occurs in individuals with SS more frequently than the general population. The most common clinical variant is that of chronic erythematous candidosis, although other types such as acute pseudomembranous candidosis may complicate SS..$^{9,10}$

Patients with overt intraoral candidosis may be completely asymptomatic. They may simply seek medical attention after developing complications such as angular chelitis, which may cause soreness on mouth opening. Angular chelitis may be due to candidal or staphylococcal infection, however, it is important to exclude other predisposing factors such as anaemia.

Patients may present with new, recurrent and atypical patterns of dental 
caries, particularly cervical caries. Factors involved may be the loss of salivary buffering capacity and the imbibition of cariogenic drinks to relieve symptoms of xerostomia. ${ }^{11}$

Even with excellent levels of oral hygiene, caries and premature tooth loss have been found in some studies where patients have a higher DMFT (decayed/ missing/filled teeth) score compared to control individuals. ${ }^{12}$ It is unclear whether patients with SS are at an increased or indeed reduced risk of periodontal attachment loss compared to a non-SS population, as current evidence is conflicting. ${ }^{13-15}$

The risk of pathological toothwear is increased in SS patients due to the reduced salivary buffering capacity. Any added risk factors such as uncontrolled gastroesophageal reflux may result in significant noncarious damage to the dentition. ${ }^{16}$

Rarely, patients may complain of symptoms of intra-oral or facial paraesthesia, or indeed anaesthesia. Specialist referral is warranted to exclude a range of aetiologies, which should also include trigeminal neuropathy. Cranial nerve neuropathies can occur in PSS, where the most common is trigeminal neuropathy. ${ }^{17}$ Trigeminal neuropathy may involve the trigeminal nerve, with a varied clinical presentation, including that of intra or extraoral numbness relating to a sensory nerve distribution.

Patients with SSS may exhibit oral manifestations related to their primary autoimmune disease. Appropriate examples include systemic lupus erythematosus (SLE), presenting with oral lichenoid lesions, which may clinically resemble oral lichen planus. Another example is scleroderma, which may present with fibrosis of the oral tissues and generalised pallor due to loss of vascularity.

\section{OCULAR MANIFESTATIONS}

The dental practitioner should be aware of the range of eye symptoms patients with SS may present with. It is also important to enquire about medical history, as systemic drugs such as antihistamines, decongestants and antidepressants can aggravate dry eye and dry mouth in SS patients. Subjective symptoms of dry eye often range from occasional discomfort to severe dryness with constant pain. The most bothersome symptoms reported by patients are dryness, grittiness, blurring of vision, itchiness and discomfort. It is important to be aware that ocular symptoms are frequently aggravated by environmental factors such as wind, central heating and air conditioning. The intensity of the ocular symptoms usually tends to get worse towards the end of the day.

Patients complaining of these eye symptoms may present with visible ocular signs such as conjunctival redness. Other patients may have no overt ocular signs but specialist ophthalmological assessment may reveal corneal and conjunctival epithelial changes. The Schirmer's test using a filter paper strip placed in the outside third of the lower eyelid has been the mainstay diagnostic tool in dry eye patients for many years.

\section{LYMPHOMA}

Malignant lymphoma was first reported in SS patients in 1963, where studies have subsequently shown PSS sufferers to have an increased risk of developing the condition. Lymphomas are solid tumours that arise in the lymphoid cells. There are two main types: Hodgkin's lymphoma (HL) and non-Hodgkin's lymphoma (NHL).

The risk of developing NHL is up to 44 times greater in PSS sufferers compared to the general population. ${ }^{18} \mathrm{~B}$-cell NHL is the most common type and recognised as a major complication of the progression of PSS. Lymphoid tissue present in the major salivary glands may be of nodal or extra-nodal origin. The majority of lymphomas that occur in SS are of extra-nodal origin arising in mucosal associated lymphoid tissue (MALT). The majority of the PSS-associated lymphoma arises from the salivary glands, with the clinical presentation being typically that of swelling. Vigilance is therefore required when dealing with PSS patients who have salivary gland swelling and/or lymphadenopathy. ${ }^{18}$ Lymphomas may also present in the oral cavity, and hence need to be included in the differential diagnosis of any intraoral swellings. ${ }^{19}$

Ultrasound may be indicated in these patients for further investigation. Disease is commonly staged using CT scanning and marrow biopsy.

\section{EXTRAGLANDULAR FEATURES}

Although glandular manifestations are the cardinal features of SS, it is important to remember that SS is a systemic disease. SS patients may present with a diffuse, firm and non-tender swelling of the salivary glands, typically of a bilateral and diffuse nature. ${ }^{20}$ Patients with SS may develop progressive major salivary gland swelling, typically being bilateral, and may be accompanied by discomfort. The swelling may be recurrent and is generally benign. The pathophysiology of such a benign swelling is that of lymphocytic infiltration of salivary tissue with associated parenchymal changes (lymphoepithelial sialadentitis). As described above, lymphomas may present in a similar manner, and appropriate investigations such as magnetic resonance imaging (MRI) of the involved glands are required to establish a correct diagnosis. ${ }^{19}$

It is important to remember that the majority of patients with SS do not have chronic major salivary gland swelling. Both these patients and even those with pre-existing swelling may develop acute suppurative sialadenitis. This may present with acute swelling, pain, regional lymphadenopathy and pyrexia and discharging pus may also be seen from the duct of the affected gland. Appropriate treatment is with a broad spectrum antibiotic.

\section{SYSTEMIC MANIFESTATIONS}

SS is not only a disease of the exocrine glands but a systemic condition.

Regarding extra-glandular symptoms and signs, fatigue is often a prominent and disabling symptom for SS patients. Myalgia and arthralgia are also common but severe inflammatory muscle or joint disease is relatively uncommon. Various forms of skin lesions secondary to disease such as vasculitis, discoid lupus and Raynaud's phenomenon can occur. Both the peripheral and central nervous system can be affected in SS but the prevalence of SS-associated neurological complications remains under debate. Peripheral neuropathy, especially involving sensory nerves, are thought to be common. Cranial nerves can be affected, leading to paraesthesia and discomfort of the face and tongue as previously described. Other organs that can be affected by SS include the lungs (interstitial pneumonitis, pleuritis), kidneys (renal tubular acidosis, interstitial glomerulonephritis), pancreas (chronic pancreatitis) and the heart (pericarditis/pericardial effusion). Preliminary data 
from the UK Primary Sjögren's Syndrome Registry (UKPSSR, www.sjogrensregistry. org) suggest that patients with PSS are at significantly increased risk of hypertension.

Serum levels of immunoglobulins, particularly IgG, are often elevated. Other haemotological and biochemical abnormalities that may be observed include lymphopaenia, thrombocytopaenia, elevated erythrocyte sedimentation rate (ESR) and raised levels of $\mathrm{C}$-reactive proteins.

Anti-Ro and Anti-La antibodies are a part of the current classification criteria for PSS. These autoantibodies are found in $60-70 \%$ of patients. ${ }^{21}$ The presence of these autoantibodies has been linked to increased disease severity and duration, as well as earlier onset of the disease. ${ }^{22}$

Finally, the symptom mostly associated with SS, xerostomia, may also influence general well-being. Patients will often report waking up at night, possibly repeatedly because of dry mouth, which consequently may result in a poor sleep pattern and onward impact in terms of fatigue and quality of life.

\section{DIAGNOSIS}

It is important that the dental practitioner has an awareness of the condition and its presentation. Most of the patients presenting with a dry mouth will not have SS. The differential diagnosis of dry mouth should, however, include:

- Sjögren's syndrome

- Drug-induced hyposalivation

- Previous head and neck radiotherapy

- Pre-existing lymphoma

- Sarcoidosis

- Hepatitis C infection

- AIDS

- Graft versus host disease.

When a clinical suspicion of SS exists, the dental practitioner should ask about eye dryness, with questions being directed appropriately. An example would be whether a patient has a gritty sensation of the eye.

As described above, SS may cause dryness of other mucosal surfaces, and hence appropriately directed questions would include the presence of pharyngeal and nasal dryness. Appropriate enquiry into any history of salivary gland swelling, fatigue, numbness or altered sensation in the head and neck would also be relevant.
Clinical examination in dental practice should involve extraoral examination of the head and neck, checking for lymphadenopathy and swelling of the major salivary glands. Intraoral examination should focus on the exclusion of any pathology, and determination of objective evidence of a dry mouth. Salivary flow rate investigations are restricted to specialist practice, and not practical in a general dental practice setting. However, sticking of the mirror to oral mucosa, lack of salivary pooling in the floor of the mouth and lack of oral mucosal wetting may give an indication of the presence of hyposalivation. If necessary, use of indices such as the clinical oral dryness score (CODS) may be helpful. ${ }^{23}$

In specialist practice, the diagnostic criteria used are the AECG criteria (American European Consensus Group). ${ }^{24}$ These criteria have six different domains (Fig. 1):

- Oral symptoms

- Ocular symptoms

- Evidence of oral signs

- Evidence of ocular dryness on examination

- Evidence of salivary gland involvement

- Histopathological analysis consequent to biopsy.

Under these criteria, particular combinations of symptoms, findings on examination, imaging and laboratory investigations are required to establish a diagnosis. For example, the presence of ocular and oral symptoms, evidence of oral and ocular dryness on examination, together with positive Anti-Ro/La autoantibodies and positive labial gland biopsy will lead to an appropriate diagnosis of SS. Labial gland biopsy is, however, an invasive investigation and associated with a risk of temporary or even permanent numbness.

In the AECG classification criteria, sialography and scintigraphy are approved imaging techniques for the objective assessment of the salivary glands. ${ }^{24}$ However, these techniques use ionising radiation and are invasive, and their popularity in the practice of oral medicine has declined in the last decade. Ultrasonographic examination of the major salivary glands has been shown to be a useful, sensitive, non-invasive and relatively inexpensive technique in the
Ocular symptoms (at least one of the following):

Daily, persistent dry eyes $>3$ months

Recurrent sensation of gritty eyes

Use of tear substitutes $>3$ times a day

\section{Oral symptoms (at least one of the following):}

Daily dry mouth $>3$ months

Recurrent or persistent salivary gland swelling

Liquid intake to aid swallowing food

Ocular signs (at least one of the following tests):

Schirmer Test (less than or equal to $5 \mathrm{~mm}$ in 5 minutes)

Rose Bengal score (less than or equal to 4)

\section{Histopathology}

Focal lymphocytic sialadenitis, with focus score less than or equal to 1 per $4 \mathrm{~mm}^{2}$ of glandular tissue

\section{Salivary gland involvement (at least one}

of the following):

Unstimulated salivary flow less than or equal to $1.5 \mathrm{ml}$ in 15 minutes

Parotid sialography showing diffuse sialectasia

Delayed uptake in salivary scintigraphy, reduced concentration and delayed excretion of tracer

\section{Autoantibodies:}

Antibodies to Ro(SSA) or La(SSB)

Fig. 1 American European Consensus Group (AECG) Revised International Classification Criteria for Sjögren's Syndrome 2002. ${ }^{24}$

assessment of patients with suspected or known SS. ${ }^{25}$ Several UK centres routinely use ultrasound in the assessment and review of SS patients. As ultrasonography is well-tolerated and easily repeatable, it is a useful tool for diagnostic work-up and follow-up assessment, although not yet recognised in the AECG criteria.

\section{MANAGEMENT}

\section{Oral aspects of treatment}

The management of SS from the perspective of the dental practitioner is that of symptomatic control, the prevention and treatment of oral complications of dry mouth. SS patients will require multidisciplinary care including early referral to oral medicine specialists to ensure delivery of oral and maxillofacial aspects of their care. ${ }^{7}$ Other factors such as poorly controlled diabetes mellitus, smoking and certain drugs that may contribute towards hyposalivation should not be overlooked.

Symptom management is particularly influenced by patient preference, with some patients preferring to take frequent sips of water or chew sugar-free chewing 
gum. Other patients supplement such measures with a range of proprietary topical products ranging from artificial saliva to salivary stimulating products. ${ }^{7}$ Saliva replacement therapy helps to lubricate the oral mucosa and aid oral clearance of food.

Examples of the more commonly prescribed artificial saliva products are Glandosane ${ }^{\circledR}$ and Saliva Orthana ${ }^{\circledR}$. Glandosane ${ }^{\circledR}$ artificial saliva spray is acidic and repeated use can result in non-carious tooth surface loss in dentate patients. Saliva Orthana ${ }^{\circledR}$ spray is not acidic, contains fluoride and is preferred to Glandosane ${ }^{\circledR}$ for use in dentate patients. The effectiveness of artificial saliva may be limited by the fact that swallowing may rapidly deplete the applied product, hence frequent application is necessary, which may be time consuming and socially unacceptable. Other products described as oral lubricants are gel-based such as Biotene Oralbalance ${ }^{\circledR}$ gel, which also contains fluoride. Topical sialogogues such as Salivix ${ }^{\circledR}$ pastilles are designed to stimulate salivary flow when sucked. As they are acidic, repeated use can result in non-carious tooth surface loss in dentate patients. ${ }^{26}$ The evidence base and limitations of the effectiveness of such products has been the subject of a recent Cochrane review. ${ }^{27}$

Pilocarpine is the only systemic drug marketed to stimulate salivary flow that is licensed in the UK. This drug is a nonselective muscarinic agonist, which in addition to stimulating the secretions of salivary and lacrimal glands, may also cause facial flushing, sweating and gastrointestinal symptoms. There is strong evidence supporting its use for the treatment of xerostomia in SS. ${ }^{26}$ The limitations of pilocarpine mainly relate to its side effect profile, which limits its use. Pilocarpine is unlikely to be effective in later stages of SS when the exocrine glands may be irreversibly damaged. ${ }^{28}$

Cevimeline is a selective muscarinic agonist with similar mode of action and side effects to pilocarpine, however, it is not licensed for use in the UK. ${ }^{27}$

The use of fluoride regimens, together with regular oral hygiene reinforcement and dietary advice are also useful measures for caries prevention (Fig. 2).

Denture wear in patients with SS may result in accelerated dental caries and periodontal disease in the dentate patient

\begin{tabular}{lll}
\hline Regimen & Example (manufacturer) & Fluoride content (parts per million) \\
\hline High fluoride toothpaste & Duraphat (Colgate) & 2,800 or 5,000 \\
\hline High fluoride daily mouthrinse & FluoriGard (Colgate) & 225 \\
\hline High fluoride varnish & Duraphat (Colgate) & 22,600 \\
\hline Remineralisation paste/foam & Tooth Mousse (GC) & 900
\end{tabular}

Fig. 2 Possible fluoride regimens with examples

and may represent an added risk factor. Appropriate advice for patients who are partially dentate and wearing dentures would be centred upon preserving their natural dentition, as outlined below. Patients with dentures may feel that their mouth is very dry and the lack of saliva prevents adequate retention of the prosthesis. In addition to advice for managing a dry mouth outlined below, a useful technique is to suggest patients lubricate their denture with an oral lubricant such as Biotene Oralbalance ${ }^{\circledR}$ gel before insertion. ${ }^{7}$ Should oral candidosis complicate SS, appropriate treatment may include topical antifungals such as oral miconazole gel, which is available in the dental practitioner's formulary, together with correction of any identifiable risk factors.

It is important that SS patients receive planned recall for routine dental examinations, which should include appropriate non-surgical periodontal treatment. It is important to appreciate that patients with SS may have associated systemic disease induced physical impairment such as rheumatoid arthritis. These patients may have difficulty attending dental appointments, and may find routine oral hygiene measures challenging.

\section{Ocular aspects of treatment}

Treatment options for SS associated dry eye are palliative. Treatments include topical medications such as artificial tear substitutes, eye gels and ointments; blockage of the draining tear ducts, that is, temporary plugs in moderate cases or permanent occlusion in more severe cases.

Tear substitutes are designed to help replace lost tear film and thus alleviate symptoms. Tear duct occlusion and other surgical procedures help reduce the loss of tears from the eye. However, their action is simply palliative as they do not treat the underlying condition, and unfortunately there are currently no therapeutic treatments available in the UK or other
European countries. Persistent use of artificial tear drops can produce a longlasting improvement in tear film stability with resolution of corneal and conjunctival epithelial changes and improvement of quality of life for all patients with SS associated dry eye.

\section{Systemic aspects of treatment}

As mentioned previously, SS is not only a disease of the exocrine glands but a systemic condition. Therefore, we recommend that all SS patients should be assessed at appropriate specialist services, which should include oral medicine/dental and maxillofacial radiology, ophthalmology and rheumatology.

The management of systemic manifestations of SS depend on the body systems involved and the severity. Immunomodulatory treatments are normally reserved for those with more severe disease, and the role of biological therapies are currently being investigated. When systemic disease is present, the treatment employed will depend on the nature of the disease. The range of therapeutic drugs that have been studied for the management of extraglandular disease ranges from conventional immunosuppressive drugs to biologic agents such as rituximab (monoclonal antibody targeting a B cell receptor). A firm evidence base for any of these agents is still lacking, however, there is increasing evidence supporting early immunomodulation of SS in an attempt to limit disease progression. ${ }^{29}$

\section{CONCLUSION}

Dental practitioners play a crucial role in the early diagnosis and management of the oral manifestations of patients with SS. It is therefore reasonable for dental practitioners to be aware of the syndrome, including an increased awareness of systemic symptoms. Indeed, as the dental practitioner will be in regular contact with this patient group as part of their routine 
recall for their dental needs, they are appropriately placed to refer this patient group to a specialist care pathway.

1. Scully C. Medical problems in dentistry. 6th ed. Edinburgh: Elsevier Health Sciences, 2010

2. Fox P C, Bowman S J, Segal B et al. Oral involvement in primary Sjögren syndrome. J Am Dent Assoc 2008: 139: 1592-1601.

3. Sood S, Anthony R, Pease C T. Sjögren's Syndrome. Clin Otolaryngol Allied Sci 2000; 25: 350-357.

4. Kurien B T,Mathews S A, Scofield R H. Can low dose diagnostic dental radiation trigger Sjögren's Syndrome? Med Hypotheses 2007; 69(5): 995-1000

5. Kassan S S, Thomas T L, Moutsopoulos H M et al. Increased risk of lymphoma in sicca syndrome. Ann Intern Med 1978; 89: 888-892.

6. Turner M, Jahangiri L, Ship J A. Hyposalivation, xerostomia and the complete denture: a systematic review. J Am Dent Assoc 2008; 139: 146-150.

7. Glore R J, Spiteri-Staines S, Paleri V. A patient with a dry mouth. Clin Otolaryngol 2009: 34: 358-363.

8. Margaix-Muñoz M, Bagán J V , Poveda R, Jiménez $Y$, Sarrión G. Sjögren's syndrome of the oral cavity. Review and update. Med Oral Patol Oral Cir Bucal 2009; 14: E325-E330.

9. Radfar L, Shea Y, Fischer S H et al. Fungal load and candidiasis in Sjögren's syndrome. Oral Surg Oral Med Oral Pathol Oral Radiol Endod 2003: 96: 283-287.

10. Yan Z, Young A L, Hua H, Xu Y. Multiple oral Candida infections in patients with Sjögren's syndrome - prevalence and clinical and drug susceptibility profiles. J Rheumato/ 2011 38: $2428-2431$
11. Wolff A, Fox P C, Porter S, Konttinen Y T. Established and novel approaches for the management of hyposalivation and xerostomia. Curr Pharm Des 2012; epub ahead of print.

12. Pederson A M, Bardow A, Nauntofte B. Salivary changes and dental caries as potential oral markers of autoimmune salivary gland dysfunction in primary Sjögren's Syndrome. BMC Clin Pathol 2005; 5: 4.

13. Antoniazzi $R$, Miranda $L A$, Zanatta $F B$ et al. Periodontal conditions of individuals with Sjögren's syndrome. J Peridontol 2009; 80: 429-435.

14. Scardina G A, Ruggieri A, Messina P. Periodontal disease and Sjögren syndrome; a possible correlation? Angiology 2009; 61: 289-293.

15. Kuru B, McCullough M J, Yilmaz S, Porter S R. Clinical and microbiological studies of periodontal disease in Sjögren syndrome patients. J Clin Periodontol 2002; 29: 92-102.

16. Wan Nik W N, Banerjee A, Moazzez R. Gastrooesophageal reflux disease symptoms and tooth wear in patients with Sjögren's syndrome. Caries Res 2011; 45: 323-326.

17. Tobón G J, Pers J O, Devauchele-Pensec V, Youinou $P$. Neurological disorders in primary Sjögren's syndrome. Autoimmune Dis 2012; 645: 967.

18. Voulgarelis M, Dafni U G, Isenberg D A, Moutsopoulos H M. Malignant lymphoma in primary Sjögren's syndrome: a multicenter, retrospective, clinical study by the European Concerted Action on Sjögren's syndrome. Arthritis Rheum 1999: 42: 1765-1772

19. Ellis $\mathrm{G} \mathrm{L}$. Lymphoid lesions of salivary glands: malignant and benign. Med Oral Patol Oral Cir Bucal 2007; 12: E479-E485.

20. Daniels T E. Sjögren's syndrome: clinical spectrum and current diagnostic controversies. Adv Dent Res 1996; 10: 3-8.

21. Hernández-Molina G, Leal-Alegre G, MichelPeregrina M. The meaning of anti-Ro and anti-La antibodies in primary Sjögren's syndrome. Autoimmun Rev 2011; 10: 123-125.

22. Bayetto K, Logan R M. Sjögren's syndrome: a review of aetiology, pathogenesis, diagnosis and management. Aust Dent J 2010; 55: 39-47.

23. Oslain S, Pramanik R, Shirodaria S, Challacombe S J Proctor G B. Investigating the relationship between hyposalivation and mucosal wetness. Oral Dis 2011 17: 109-114.

24. Vitali C, Bombardieri $\mathrm{S}$, Jonsson $\mathrm{R}$ et al. Classification criteria for Sjögren's syndrome: a revised version of the European criteria proposed by the American-European Consensus Group. Ann Rheum Dis 2002; 61: 554-558.

25. Greenwood M, Seymour R A, Meechan J G. Textbook of human disease in dentistry. Chichester: WileyBlackwell, 2009

26. Brennan M T, Shariff G, Lockhart P B, Fox P C Treatment of xerostomia: a systematic review of therapeutic trials. Dent Clin North Am 2002 46: 847-856.

27. Furness $S$, Worthington $H V$, Bryan $G$, Birchenough S, McMillan R. Interventions for the management of dry mouth: topical therapies. Cochrane Database Syst Rev 2011; 7: CD008934.

28. Fox P C. Salivary enhancement therapies. Caries Res 2004; 38: 241-246.

29. Ramos-Casals M, Brito-Zerón P, Sisó-Almirall A, Bosch X, Tziofas A G. Topical and systemic medications for the treatment of primary Sjögren's syndrome. Nat Rev Rheumatol 2012; 8: 399-411. 